\title{
Identification of potential gene signatures associated with osteosarcoma by integrated bioinformatics analysis
}

\author{
Yutao Jia ${ }^{1}$, Yang Liu ${ }^{1}$, Zhihua Han ${ }^{\text {Corresp., }}{ }^{2}$, Rong Tian ${ }^{1}$ \\ ${ }^{1}$ Department of Spine Surgery, Tianjin Union Medical Center, Tianjin, China \\ 2 Department of Anesthesiology, Tianjin Union Medical Center, Tianjin, China \\ Corresponding Author: Zhihua Han \\ Email address: hanzhihua1996@163.com
}

Background. Osteosarcoma (OS) is the most primary malignant bone cancer in children and adolescents with a high mortality rate. This work aims to screen novel potential gene signatures associated with OS by integrated microarray analysis of Gene Expression Omnibus (GEO) database. Material and Methods. The OS microarray datasets were searched and downloaded from GEO database to identify differentially expressed genes (DEGs) between OS and normal samples. Afterwards, the functional enrichment analysis, protein-protein interaction (PPI) network analysis and transcription factor (TF)-target gene regulatory network were applied to uncover the biological function of DEGs. Finally, two published OS datasets (GSE39262 and GSE126209) were obtained from GEO database for evaluating the expression level and diagnostic values of key genes. Results. Totally, 1059 DEGs (569 up-regulated DEGs and 490 down-regulated DEGs) between OS and normal samples were screened. Functional analysis showed that these DEGs were markedly enriched in $214 \mathrm{GO}$ terms and 54 KEGG pathways such as pathways in cancer. Five genes (CAMP, METTL7A, TCN1, LTF and CXCL12) acted as hub genes in PPI network. Besides, METTL7A, CYP4F3, TCN1, LTF and NETO2 were key genes in TF-gene network. Moreover, Pax-6 regulated four key genes (TCN1, CYP4F3, NETO2 and CXCL12). The expression levels of four genes (METTL7A, TCN1, CXCL12 and NETO2) in GSE39262 set were consistent with our integration analysis. The expression levels of two genes (CXCL12 and NETO2) in GSE126209 set were consistent with our integration analysis. ROC analysis of GSE39262 set revealed that CYP4F3, CXCL12, METTL7A, TCN1 and NETO2 had good diagnostic values for OS patients. ROC analysis of GSE126209 set revealed that CXCL12, METTL7A, TCN1 and NETO2 had good diagnostic values for OS patients. 
1

2

3 Yutao Jia ${ }^{1}$, Yang Liu ${ }^{1}$, Zhihua Han ${ }^{2 \#}$, Rong Tian ${ }^{1}$

$4{ }^{1}$ Department of Spine Surgery, Tianjin Union Medical Center, Tianjin, China;

5 2. Department of Anesthesiology, Tianjin Union Medical Center, Tianjin, China;

\section{7 \#Correspondence to:}

8 Zhihua Han;

9 Address: Department of Anesthesiology, Tianjin Union Medical Center; No.190, Jieyuan Road,

10 Hongqiao District, Tianjin 300121, China;

11 Tel: $(+86) 022-27557374$

12 Email: hanzhihua1996@163.com 


\section{Abstract}

Background. Osteosarcoma (OS) is the most primary malignant bone cancer in children and adolescents with a high mortality rate. This work aims to screen novel potential gene signatures associated with OS by integrated microarray analysis of Gene Expression Omnibus (GEO) database.

Material and Methods. The OS microarray datasets were searched and downloaded from GEO database to identify differentially expressed genes (DEGs) between OS and normal samples.. Afterwards, the functional enrichment analysis, protein-protein interaction (PPI) network analysis and transcription factor (TF)-target gene regulatory network were applied to uncover the biological function of DEGs. Finally, two published OS datasets (GSE39262 and GSE126209) were obtained from GEO database for evaluating the expression level and diagnostic values of key genes.

Results. Totally, 1059 DEGs (569 up-regulated DEGs and 490 down-regulated DEGs) between OS and normal samples were screened. Functional analysis showed that these DEGs were markedly enriched in 214 GO terms and 54 KEGG pathways such as pathways in cancer. Five genes (CAMP, METTL7A, TCN1, LTF and CXCL12) acted as hub genes in PPI network. Besides, METTL7A, CYP4F3, TCN1, LTF and NETO2 were key genes in TF-gene network. Moreover, Pax-6 regulated four key genes (TCN1, CYP4F3, NETO2 and CXCL12). The expression levels of four genes (METTL7A, TCN1, CXCL12 and NETO2) in GSE39262 set were consistent with our integration analysis. The expression levels of two genes (CXCL12 and NETO2) in GSE126209 set were consistent with our integration analysis. ROC analysis of GSE39262 set revealed that CYP4F3, CXCL12, METTL7A, TCN1 and NETO2 had good diagnostic values for OS patients. ROC analysis of GSE126209 set revealed that CXCL12, METTL7A, TCN1 and NETO2 had good diagnostic values for OS patients.

Conclusion. Four genes (CXCL12, METTL7A, TCN1 and NETO2) may be involved in the development of OS, which will contribute to develop novel therapeutic strategies for OS management. 
45 Keywords: Osteosarcoma, Diagnosis, Genes, Activating transcription factor, Bioinformatic. 46 
47

48

49

50

51

52

\section{Introduction}

Osteosarcoma (OS) is a type of primary malignant bone cancer that causes public health concern, especially in children and adolescents (Isakoff MS 2015; Lindsey et al. 2017). Several treatment strategies of OS such as surgical resection, traditional adjuvant chemotherapy and radiotherapy have been favored by clinical oncologists in the past few decades (Nagarajan et al. 2011). Accordingly, the 5-year survival rate has been raised to approximately 70\% (Bielack SS 2002). However, it is estimated that $80 \%$ of OS patients may suffer from the micro-metastasis, which cannot be detected at early diagnosis (Messerschmitt et al. 2009). Although multiple methods for the diagnosis and treatment of OS have been developed, new methods for the prevention and treatment of OS are still needed. The pathogenesis of OS progression remains not fully understood. Therefore, the identification of effective diagnostic makers and exploring underlying molecular etiology of OS is a pressing need.

The emergence of high-throughput sequencing technology has become an effective way to illuminate the pathogenic genes in a variety of human diseases, which help to explore pathogenesis and develop biomarkers. Many research groups have screened multiple biomarkers associated with OS by analyzing gene expression data. For example, Sun et al evaluated the difference of genes in the expression level between OS metastasis and OS non-metastasis and discovered that $T G F B 1, L F T 3, K D M 1 A$, and $K R A S$ may participate in the occurrence of OS. Xiong et al found that CCT3, COPS3 and WWP1 were involved in the OS development by integrating gene expression and genomic aberration data (Xiong Y 2015). Liu et al constructed a co-expression network based on a Gene Expression Omnibus (GEO) dataset and identified many potential biomarkers such as CTLA4 and PBF for diagnosis and treatment of OS (Liu X 2017). However, the molecular mechanisms of OS initiation and development have not been fully explored.

In this study, we retrieved GEO database and obtained four OS datasets. Subsequently, the differentially expressed genes (DEGs) between OS samples and normal samples were obtained and subjected to functional analysis. A protein-protein interaction (PPI) network was constructed 
followed by the establishment of transcription factor (TF)-target regulatory network. Following this, we downloaded two published GEO datasets of OS as the validation set for assessing the expression levels of key candidate genes. Finally, the receiver operating characteristic (ROC) analysis was conducted to evaluate the diagnostic values of key candidate genes. This study will discover novel gene signatures associated with OS, providing new trains of thought for the diagnosis and treatment of OS.

\section{Materials and methods}

Data acquisition

The datasets were retrieved from the National Center for Biotechnology Information-GEO (http://www.ncbi.nlm.nih.gov/geo/) repository using the key terms of 'osteosarcoma' AND 'Homo sapiens'[porgn]. All selected datasets in this study should meet the following criteria: i ) the datasets contained genome-wide expression data of tumor tissues and normal control tissues of OS patients; and ii ) all data were standardized or raw data. As shown in table S1, a total of five datasets were obtained. Notably, the GSE9508 dataset contained over 50\% missing data and was subsequently removed from the following analysis. Eventually, four datasets (GSE12865, GSE19276, GSE87624 and GSE99671) were included in this study, which included 118 OS tissues and 28 normal bone tissues. The GSE12865 series (GPL6244 platform) included a total of 14 samples (12 OS and 2 normal control tissues). The platform for GSE19276 was GPL6848 including 44 OS and 5 normal control bone tissue. The platform for GSE87624, consisting of 44 OS and 3 normal control bone tissue samples, was GPL11154. GSE99671 was in GPL20148 platform, which contained 18 OS and 18 normal control bone tissue samples. The platform and series matrix files were downloaded. The dataset information was listed in table S1. The impact of different platforms on the results, we normalized the data through the log function, and centralized and standardized the scale function to eliminate the impact of the dimension on the data structure.

Data pre-processing and DEGs identification

The standardized data from included datasets were firstly processed as follows: i ) the probes 
101 that mapped to several genes were deleted; and ii ) if the gene was matched by multiple probes,

102 the probe with the greatest gene expression value would be retained. Overall, there were 103 overlapping 14981 genes among four datasets. Subsequently, MetaMA (https://cran.r104 project.org/web/packages/metaMA/), an R package, was used to combine data from four GEO 105 datasets. Individual p-values were obtained using Limma R package. The inverse normal method

106

107

108

109

110

111

112

113

114

115

116

117

118

119

120

121

122

123

124

125

126

127 was used to combine $P$ values in meta-analysis (Marot G 2009). We carried out the multiple comparison correction by Benjamini \& Hochberg approach to acquire false discovery rate (FDR). Herein, the DEGs between OS tissues and normal controls were defined according to the cutoff of false discovery rate $($ FDR $)<0.05$ and those DEGs with different directionality were removed from this study. Finally, the hierarchical clustering analysis of top 100 DEGs was also carried out by pheatmap package in R software.

Functional enrichment analyses

To systematically explore the underlying biological functions of identified DEGs, the Metascape (http://metascape.org/gp/index.html), an online tool that integrates multiple data resources such as Gene Ontology (GO), Kyoto Encyclopedia of Genes and Genomes (KEGG) and Universal Protein (Uniprot) database, was used to perform GO and KEGG pathway enrichment analysis of DEGs. GO analysis involved three categories: biological process (BP), cellular component (CC) and molecular function $(\mathrm{MF}) . P$ values $<0.05$ was set as the thresholds for significant enrichment analyses.

\section{Protein-protein interaction (PPI) network analysis}

The Search Tool for the Retrieval of Interacting Genes/Proteins (STRING) database, a freely web-based analytic tool, can predict the interactions among proteins (Szklarczyk et al. 2019). Here, a PPI analysis was conducted to examine the interactive associations between protein products of DEGs. The Cytoscape software (http://www.cytoscape.org) was utilized to establish a PPI network. In addition, the CytoNCA (http://apps.cytoscape.org/apps/cytonca) was used to analyze topological characteristics of PPI network. The top 15 nodes were considered as hub genes according to the degree value.

Peer) reviewing PDF | (2020:06:50311:3:0:CHECK 29 Mar 2021) 


\section{TF-candidate gene network analysis}

TFs can bind to specific DNA sequences in promoter region of target gene to regulate gene expression. The top 20 up- and down-regulated genes were regarded as the candidate genes. The DNA sequences $(2 \mathrm{~kb})$ in the upstream promoter region of these candidate genes were firstly downloaded from the University of California, Santa Cruz (http://www.genome.ucsc.edu/) databases. Then, we employed online match tool from TRANSFAC (http://genexplain.com/transfac) to predict potential TFs that targeted candidate genes. Notably, the TFs that had only one binding site with target genes were retained in this study. Finally, the Cytoscape software was used to build a transcriptional regulatory network and perform node degree analysis.

Evaluation the expression level and diagnostic values of key genes

Two published OS datasets were obtained from GEO database for the expression level evaluation of seven key DEGs (CAMP, METTL7A, TCN1, LTF, CXCL12, CYP4F3 and NETO2). Then, we performed a ROC analysis using the pROC package in $\mathrm{R}$ software (http://web.expasy.org/pROC/) to evaluate the diagnostic value of these seven DEGs. Accordingly, the area under the curve (AUC) was computed and the ROC curve was built. The AUC value $>0.8$ showed a good diagnostic value for OS.

\section{Results}

\section{Identification of DEGs}

After data pre-processing, a total of 1059 DEGs (569 up-regulated genes and 490 downregulated genes) were identified between OS samples and normal controls according to methods described above. The clustering analysis indicated that top 100 DEGs could distinguish OS samples and controls from four datasets (Figure S1). The top 20 up- and down-regulated genes were listed in table 1.

\section{$G O$ and KEGG enrichment analysis of DEGs}

The GO enrichment analysis of DEGs showed that a total of $214 \mathrm{GO}$ terms were enriched, including 194 GO-BP terms, 15 GO-CC terms and 5 GO-MF terms (Table S1). Specifically, for 
155

156

157

158

159

160

161

162

163

164

165

166

167

168

169

170

171

172

173

174

175

176

177

178

179

180

181

GO-BP analysis, these DEGs were strongly associated with positive regulation of cell death, myeloid cell activation involved in immune response and regulation of protein kinase activity. Meanwhile, protein domain specific binding and transcription factor binding were significantly enriched GO-MF terms. Many DEGs were primarily involved in multiple GO-CC terms, such as anchored component of membrane and tertiary granule. The top 20 clusters of significantly enriched GO terms were displayed in figure 1. In addition, these DEGs were markedly enriched in 20 KEGG pathways such as regulation of lipolysis in adipocytes, protein processing in endoplasmic reticulum and pathways in cancer (Table 2). Notably, the top 20 up-regulated genes did not enrich in any KEGG pathway. However, four of top 20 down-regulated genes played vital roles in multiple significantly enriched KEGG pathways, including FABP4 (fatty acid binding protein 4), CXCL12 (C-X-C motif chemokine ligand 12), CXCL12 (C-X-C motif chemokine ligand 12) and $C A T$ (Catalase). More specifically, FABP4 was involved in regulation of lipolysis in adipocytes and CXCL12 participated in pathways in cancer and axon guidance (Table 2). CYP4F3 was closely correlated with arachidonic acid metabolism pathway and $C A T$ was significantly enriched in biosynthesis of amino acids and AMPK signaling pathway (Table 2).

\section{PPI network analysis}

To determine the relationships among DEGs, a PPI network was built based on the STRING database, which included 109 nodes and 196 protein pairs (Figure 2). The top 15 hub genes contain $P P B P$ (pro-platelet basic protein; degree $=13$ ), $C A M P$ (cathelicidin antimicrobial peptide, degree $=13$ ), LTF (lactotransferrin, degree $=12$ ), BST1 (bone marrow stromal cell antigen 1, degree $=12), C X C R 2(\mathrm{C}-\mathrm{X}-\mathrm{C}$ motif chemokine receptor 2, degree = 10), OLFM4 $($ olfactomedin 4, degree $=10)$, STOM $($ stomatin, degree $=10), T C N 1($ transcobalamin 1, degree $=$ 9), SLC4A1 (solute carrier family 4 member 1, degree = 9), LTA4H (leukotriene A4 hydrolase, degree =9), S100A9 (S100 calcium binding protein A9, degree = 9), CXCL12 (degree = 8), CLEC12A (C-type lectin domain family 12 member A, degree = 8), RAB37 (RAB37, member RAS oncogene family, degree $=8$ ) and METTL7A (methyltransferase like 7A, degree = 8). More 
182 notably, these genes were all down-regulated.

183 TF-target network analysis

$184 \mathrm{TF}$ exerts crucial roles in regulating the expression of target gene. Herein, we employed 185 TRANSFAC to predict TFs that regulated 20 up-regulated and down-regulated DEGs. As shown 186 in Figure 3, the TF-target gene regulatory network contained 95 nodes (55 TFs and 40 genes) 187 and 275 TF-gene pairs. The top 15 genes in TF-target network were CHL1 (cell adhesion 188 molecule L1 like, down-regulation, degree = 16), SERPINB2 (serpin family B member 2, down189 regulation, degree $=15$ ), SLC28A3 (solute carrier family 28 member 3, down-regulation, degree $190=11$ ), ZC3H8 (zinc finger CCCH-type containing 8, up-regulation, degree $=10$ ), LAPTM4B 191 (lysosomal protein transmembrane 4 beta, up-regulation, degree $=10$ ), DYRK4 (dual specificity 192 tyrosine phosphorylation regulated kinase 4, up-regulation, degree $=9$ ), METTL7A (solute carrier 193 family 28 member 3, down-regulation, degree =9), KCNG1 (potassium voltage-gated channel modifier subfamily $\mathrm{G}$ member 1, down-regulation, degree = 9), $C Y P 4 F 3$ (down-regulation, degree $=9)$, GNL2 (G protein nucleolar 2, up-regulation, degree = 8), HBD (hemoglobin subunit delta, down-regulation, degree = 8), TCN1 (down-regulation, degree = 8), ZZZ3 (zinc finger ZZtype containing 3, up-regulation, degree $=8$ ), NETO2 (neuropilin and tolloid like 2, upregulation, degree $=8$ ), and $L T F$ (lactotransferrin, down-regulation, degree $=8$ ). In addition, the top 6 TFs that covered the most downstream genes were exhibited in table 3, which contained Pax-4, 1-Oct, Nkx2-5, HNF-4, FOXD3 and Pax-6. Interestingly, TCN1, CYP4F3, NETO2 and CXCL12 were regulated by Pax-6 (Table 3).

Evaluation the expression level and diagnostic values of key genes

An external dataset (GSE39262) was obtained from GEO database, which contained 10 human osteosarcoma cell lines and 5 untransformed cell lines samples. The platform for this dataset was GPL96 [HG-U133A] Affymetrix Human Genome U133A Array. GSE126209 was downloaded from GEO database, which inclued 12 osteosarcomas tumors and 11 adjacent normal tissues samples. The platform for this dataset was GPL20301 Illumina HiSeq 4000. Seven key genes (CAMP, METTL7A, TCN1, LTF, CXCL12, CYP4F3 and NETO2) were selected to verify in 
209

210

211

212

213

214

215

216

217

218

219

220

221

222

223

224

225

226

227

228

229

230

231

232

233

234

235

GSE39262. Among them, CAMP, METTL7A, TCN1, LTF and CXCL12 acted as hub genes in PPI network. METTL7A, CYP4F3, TCN1, LTF and NETO2 were key genes in TF-gene network. Moreover, Pax-6 regulated four key genes (TCN1, CYP4F3, NETO2 and CXCL12). The gene differential expression analysis of GSE39262 dataset revealed that NETO2 was significantly upregulated while $C X C L 12, M E T T L 7 A$ and TCN1 were significantly down-regulated, which were consistent with our integration analysis (Figure 4; Table S2). The gene differential expression analysis of GSE126209 dataset displayed that NETO2 was significantly up-regulated while CXCL12 was significantly down-regulated, which were consistent with our integration analysis (Figure 5; Table S3). ROC analysis is a commonly used method to evaluate the value of genetic diagnosis and has been used in previously biomedical works (Le et al. 2019; Le et al. 2019; Thi et al. 2020). Additionally, the results of GSE39262 dataset showed that five genes had good diagnostic values for OS (CXCL12, CYP4F3, METTL7A, NETO2 and TCN1; Figure 6). The AUC of CXCL12 was 1.000 and the specificity and sensitivity of this model were $100.0 \%$ and $100 \%$, respectively. The AUC of $C Y P 4 F 3$ was 0.840 and the specificity and sensitivity of this model were $80.0 \%$ and $80.0 \%$, respectively. The AUC of METTL7A was 0.900 and the specificity and sensitivity of this model were $100.0 \%$ and $70.0 \%$, respectively. The AUC of NETO2 was 0.860 and the specificity and sensitivity of this model were $100.0 \%$ and $80.0 \%$, respectively. The AUC of TCN1was 0.820 and the specificity and sensitivity of this model were $80.0 \%$ and $90.0 \%$, respectively. Ultimately, the results of GSE126209 dataset showed that four genes had good diagnostic values for OS (CXCL12, METTL7A, NETO2 and TCN1; Figure 7). CXCL12 was 1.000 and the specificity and sensitivity of this model were $100.0 \%$ and $100 \%$, respectively. The AUC of METTL7A was 0.856 and the specificity and sensitivity of this model were $100.0 \%$ and $83.3 \%$, respectively. The AUC of NETO2 was 0.833 and the specificity and sensitivity of this model were $100.0 \%$ and $75.0 \%$, respectively. The AUC of TCN1was 0.879 and the specificity and sensitivity of this model were $81.8 \%$ and $83.3 \%$, respectively.

\section{Discussion}

OS is a common malignant bone tumor and originates from mesenchymal stromal cells (MSCs) 
236 (Xiao W 2013). The heterogeneous histopathological characteristics and complex genomic 237 landscape of OS have been major challenges for elaborating underlying the molecular 238 pathogenesis of OS. In this study, we included four OS datasets and identified 1059 DEGs (569 239 up-regulated DEGs and 490 down-regulated DEGs) between OS and normal samples. These genes were significantly enriched in 54 KEGG pathways such as pathways in cancer. Moreover, CAMP, METTL7A, TCN1, LTF and CXCL12 served as hub genes in PPI network. METTL7A, CYP4F3, TCN1, LTF and NETO2 were key players in TF-target gene regulatory network. Interestingly, TCN1, CYP4F3, NETO2 and CXCL12 were all regulated by Pax-6. Additionally, the expression patterns of key genes (CAMP, METTL7A, TCN1, LTF, CXCL12, CYP4F3 and NETO2) were selected to verify in two published OS datasets (GSE39262 and GSE126209). $C A M P$, also known as $h C A P 18$ or $L L 37$, is an antimicrobial peptide gene in human (Larrick et al. 1995). The C-terminal of the protein product of $C A M P$ contains a 37-amino acid-long peptide with broad spectrum-antibacterial activity (Vandamme D 2012). There are positive expressions of $C A M P$ in the multiple cell systems, such as epithelial cells, neutrophils and macrophages (Dhawan P 2015; Frew L 2014; Li Y 2018). Wu et al suggested that bone marrow stroma could express $C A M P$, which may be a potential ex vivo priming factor for hematopoietic stem progenitor cells to promote hematopoietic reconstitution after transplantation (Wu et al. 2012). Later, Coffelt et al discovered that CAMP expression level was elevated in MSCs compared to that in ovarian cancer cells (Coffelt SB 2019). Herein, our analysis showed that CAMP was the most down-regulated gene in patients suffering from OS. Besides, $C A M P$ acted as a hub gene in PPI network, suggesting that this gene may be involved in the pathologic mechanism of OS. Although the underlying role of CAMP on the initiation and progression of OS has not been investigated, available evidence showes that $C A M P$ plays significant roles in several cancers, including breast cancer, lung cancer and pancreatic cancer (García-Quiroz J 2016; Sainz et al. 2015; von Haussen et al. 2008). More notably, existing data indicated that $C A M P$ had either carcinogenic or anti-cancer effects (Chen X 2018; Wu WK 2010). Therefore, the influence of $C A M P$ on OS occurrence and development needs to be further clarified in future. 
263 Our gene differential expression revealed that CXCL12 and TCN1 were down-regulated in OS 264 patients, which were verified in a validation dataset. Moreover, these two genes also acted as hub 265 genes in PPI network. In addition, up-regulated NETO2 and down-regulated CYP4F3 had high 266 degree in TF-gene regulatory network. Interestingly, CXCL12, TCN1, NETO2 and CYP4F3, 267 regulated by Pax-6, exhibited important diagnostic values for OS. CXCL12 is also called stromal 268 cell-derived factor-1 (SDF-1) and can bind to G-protein-coupled chemokine receptor CXCR4 (T 269 2014). Increasing studies suggested that $C X C L 12 / C X C R 4$ axis played pivotal roles in tumor 270 growth and development (F 2004; Lu Y 2015; Perissinotto E 2005). Li et al highlighted that epigenetic regulation of CXCL12 by DNA methyltransferase 1 was associated with the metastasis and immune response in OS (Li B 2018). Previous reports also indicated that down-

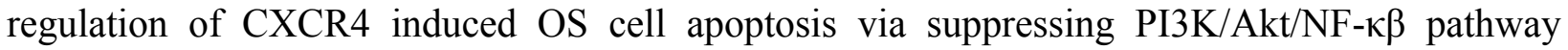
(Pollino S 2019). However, there is no directive evidence to support the involvement of TCN1, NETO2 and CYP4F3 in OS. Notably, Pax-6 is a highly conserved evolutionarily TF and belongs to paired box TF family (Mansouri A 1996). Several studies have pointed out that Pax6 participated in the regulation of cancer cell proliferation and progression (Shyr et al. 2010; Zong $\mathrm{X}$ 2011). Yang et al established a TF-top 20 DEGs regulatory network by integrating and analyzing three GEO datasets (GSE66673, GSE49003 and GSE37552), and found that Pax-6 down-regulated BMP6 expression in non-metastatic OS samples (Yang C 2019). Taken together, we inferred that $C X C L 12 / T C N 1 / N E T O 2 / C Y P 4 F 3-P a x-6$ axis may be implicated in the pathogenesis of OS, and four genes (CXCL12, TCN1, NETO2 and CYP4F3) were novel diagnostic biomakers for OS.

METTL7A and LTF are reported to act as tumor suppressor genes (Qi et al. 2017; Zhang et al. 2011; Zhang et al. 2015). Similarly, our findings showed that the expressions of METTL7A and LTF were decreased in OS samples. Moreover, these two genes were both hub genes in PPI analysis and key gene nodes in TF-gene regulatory analysis. These results implied that METTL7A and LTF may be correlated with underlying mechanisms of OS. However, the potential effects of METTL7A and LTF down-regulation on OS progression needs to be further 
290 investigated.

291 Although we have identified multiple novel gene signatures associated with OS, there are still

292 limitations in this work. Our conclusion was drawn based on an integrated bioinformatic analysis.

293 Therefore, additional experiments are required to confirm our findings. In addition, a larger 294 sample size verification will also improve the reliability of our conclusion. Moreover, the clinical 295 information should be collected to evaluate the diagnostic value of biomarkers for OS patients.

296 Finally, the biological significances of key biomarkers will be investigated in model systems or 297 cell lines.

298 In summary, a total of 1059 DEGs were identified between OS and normal samples. Among 299 them, up-regulation of NETO2 and down-regulation of METTL7A, TCN1, and CXCL12 may be 300 potential gene signatures related to OS. Pax-6 was also probably associated with the pathological 301 process of OS. However, a comprehensive bioinformatics analysis with larger sample size and in 302 vivo or in vitro assays should be performed to confirm our results.

303 Acknowledgement

304 Not applicable.

\section{Competing interests}

306 The authors declare that they have no known competing financial interests or personal 307 relationships that could have appeared to influence the work reported in this paper. 
311

312

313

314

315

316

317

318

319

320

321

322

323

324

325

326

327

328

329

330

331

\section{References}

Bielack SS K-BB, Delling G, Exner GU, Flege S, Helmke K, Kotz R, Salzer-Kuntschik M, Werner M, Winkelmann W, Zoubek A, Jürgens H, Winkler K. 2002. Prognostic factors in high-grade osteosarcoma of the extremities or trunk: an analysis of 1,702 patients treated on neoadjuvant cooperative osteosarcoma study group protocols. J Clin Oncol 20:776-790.

Chen X ZX, Qi G, Tang Y, Guo Y, Si J, Liang L. 2018. Roles and Mechanisms of Human Cathelicidin LL-37 in Cancer. Cell Physiol Biochem 47:1060-1073.

Coffelt SB MF, Watson K, Zwezdaryk KJ, Dembinski JL, LaMarca HL, Tomchuck SL, Honer zu Bentrup K, Danka ES, Henkle SL, Scandurro AB. 2019. The pro-inflammatory peptide LL-37 promotes ovarian tumor progression through recruitment of multipotent mesenchymal stromal cells. Proc Natl Acad Sci U S A 106:3806-3811.

Dhawan P WR, Sun C, Gombart AF, Koeffler HP, Diamond G, Christakos S. 2015. C/EBP $\alpha$ and the Vitamin D Receptor Cooperate in the Regulation of Cathelicidin in Lung Epithelial Cells. J Cell Physiol 230:464-472.

F B. 2004. Cancer and the chemokine network. Nat Rev Cancer 4:540-550.

Frew L MS, McKinlay AT, McHugh BJ, Doust A, Norman JE, Davidson DJ, Stock SJ. 2014. Human cathelicidin production by the cervix. PLoS One 9:e103434.

García-Quiroz J G-BR, Santos-Martínez N, Avila E, Larrea F, Díaz L. 2016. Calcitriol stimulates gene expression of cathelicidin antimicrobial peptide in breast cancer cells with different phenotype. J Biomed Sci 23:78. 
332 Isakoff MS BS, Meltzer P, Gorlick R. 2015. Osteosarcoma: Current Treatment and a 333 Collaborative Pathway to Success. J Clin Oncol 33:3029-3035.

334 Larrick J, Hirata M, Balint RF, Lee J, Zhong J, and Wright S. 1995. Human CAP18: a novel antimicrobial lipopolysaccharide-binding protein. Infect Immun 63:1291-1297.

Le N Q K, Yapp E K Y, H. Y. Yeh. 2019. ET-GRU: using multi-layer gated recurrent units to identify electron transport proteins. BMC Bioinformatics 20.1.

Le N Q K, Yapp, E.K. Y, Nagasundaram N, Chua, M C H,Yeh H. 2019. Computational identification of vesicular transport proteins from sequences using deep gated recurrent units architecture. Computational and Structural Biotechnology Journal 17.

Li B WZ, Wu H, Xue M, Lin P, Wang S, Lin N, Huang X, Pan W, Liu M, Yan X, Qu H, Sun L, Regulation of CXCL12 Plays a Critical Role in Mediating Tumor Progression and the Immune Response In Osteosarcoma. Cancer Res 78:3938-3953.

Li Y ØS, Johnsen IB. 2018. Human Metapneumovirus Infection Inhibits Cathelicidin Antimicrobial Peptide Expression in Human Macrophages. Front Immunol 9:902. $4: 25-43$.

Liu X HA, Zhao JL, Chen FL. 2017. Identification of Key Gene Modules in Human Osteosarcoma by Co-Expression Analysis Weighted Gene Co-Expression Network Analysis (WGCNA). J Cell Biochem 118:3953-3959. 
353

354

355

356

357

358

359

360

361

362

363

364

365

366

367

368

369

370

371

372

373

SDF-1/CXCR4 promotes F5M2 osteosarcoma cell migration by activating the $\mathrm{Wnt} / \beta$ catenin signaling pathway. Med Oncol 32:194.

Mansouri A HM, Gruss P. 1996. Pax genes and their roles in cell differentiation and development. Curr Opin Cell Biol 8:851-857.

Marot G FJ, Mayer CD, Jaffrézic F. 2009. Moderated effect size and p-value combinations for microarray meta-analyses. Bioinformatics 25:2692-2699.

Messerschmitt PJ, Garcia RM, FW A-K, EM G, and Getty PJ. 2009. Osteosarcoma. J Am Acad Orthop Surg 17:515-527.

Nagarajan R, Kamruzzaman A, Ness KK, Marchese VG, Sklar C, Mertens A, Yasui Y, Robison LI, and Marina N. 2011. Twenty years of follow-up of survivors of childhood osteosarcoma: a report from the Childhood Cancer Survivor Study. Cancer 117:625-634.

Perissinotto E CG, Leone F, Fonsato V, Mitola S, Grignani G, Surrenti N, Sangiolo D, Bussolino F, Piacibello W, Aglietta M. 2005. Involvement of chemokine receptor 4/stromal cellderived factor 1 system during osteosarcoma tumor progression. Clin Cancer Res 11:490497.

Pollino S PE, Dozza B, Bientinesi E, Piccinni-Leopardi M, Lucarelli E, Righi A, Benassi MS, Pazzaglia L. 2019. CXCR4 in human osteosarcoma malignant progression. The response of osteosarcoma cell lines to the fully human CXCR4 antibody MDX1338. J Bone Oncol $17: 100239$.

Qi L, Song Y, Chan THM, Yang H, Lin CH, Tay DJT, Hong H, Tang SJ, Tan KT, Huang XX, Lin JS, Ng VHE, Maury JJP, Tenen DG, and Chen L. 2017. An RNA editing/dsRNA 
374

375

376

377

378

379

380

381

382

383

384

385

386

387

388

389

390

391

392

393

394

binding-independent gene regulatory mechanism of ADARs and its clinical implication in cancer. Nucleic Acids Res 45:10436-10451.

Sainz B, Jr., Alcala S, Garcia E, Sanchez-Ripoll Y, Azevedo MM, Cioffi M, Tatari M, MirandaLorenzo I, Hidalgo M, Gomez-Lopez G, Cañamero M, Erkan M, Kleeff J, García-Silva S, Sancho P, Hermann PC, and Heeschen C. 2015. Microenvironmental hCAP-18/LL-37 promotes pancreatic ductal adenocarcinoma by activating its cancer stem cell compartment. Gut 64:1921-1935.

Shyr C, Tsai MY, Yeh S, HY K, YC C, Wong Pl, CC H, KE H, and Chang C. 2010. Tumor suppressor PAX6 functions as androgen receptor co-repressor to inhibit prostate cancer growth. Prostate 70:190-199.

Szklarczyk D, Gable AL, Lyon D, Junge A, Wyder S, Huerta-Cepas J, Simonovic M, Doncheva NT, Morris JH, Bork P, Jensen LJ, and Mering Cv. 2019. STRING v11: protein-protein association networks with increased coverage, supporting functional discovery in genome-wide experimental datasets. Nucleic acids research 47:D607-D613. 10.1093/nar/gky1131

Thi D, Trang L, Khanh L. 2020. Using deep neural networks and biological subwords to detect protein S-sulfenylation sites. Briefings in Bioinformatics.

T N. 2014. CXC chemokine ligand 12 (CXCL12) and its receptor CXCR4. J Mol Med (Berl) $92: 433-439$.

Vandamme D LB, Luyten W, Schoofs L. 2012. A comprehensive summary of LL-37, the factotum human cathelicidin peptide. Immunol 280:22-35. 
395

396

397

398

399

400

401

402

403

404

405

406

407

408

409

410

411

412

413

414

415

von Haussen J, Koczulla R, Shaykhiev R, Herr C, Pinkenburg O, Reimer D, Wiewrodt R, Biesterfeld S, Aigner A, Czubayko F, and Bals R. 2008. The host defence peptide LL37/hCAP-18 is a growth factor for lung cancer cells. Lung Cancer 59:12-23.

Wu W, Kim CH, Liu R, Kucia M, Marlicz W, Greco N, Ratajczak J, MJ L, and Ratajczak M. 2012. The bone marrow-expressed antimicrobial cationic peptide LL-37 enhances the responsiveness of hematopoietic stem progenitor cells to an SDF-1 gradient and accelerates their engraftment after transplantation. Leukemia 26:736-745.

Wu WK WG, Coffelt SB, Betancourt AM, Lee CW, Fan D, Wu K, Yu J, Sung JJ, Cho CH. 2010. Emerging roles of the host defense peptide LL-37 in human cancer and its potential therapeutic applications. Int J Cancer 127:1741-1747.

Xiao W MA, Hogendoorn PC, Cleton-Jansen AM. 2013. Mesenchymal stem cell transformation and sarcoma genesis. Clin Sarcoma Res 3:10.

Xiong Y WS, Du Q, Wang A, Wang Z. 2015. Integrated analysis of gene expression and genomic aberration data in osteosarcoma (OS). Cancer Gene Ther 22:524-529.

Yang C HD, Ma C, Ren J, Fu L, Cheng C, Li B, Shi X. 2019. Identification of Pathogenic Genes and Transcription Factors in Osteosarcoma. Pathol Oncol Res.

Zhang H, Feng X, Liu W, Jiang X, Shan W, Huang C, Yi H, Zhu B, Zhou W, Wang L, Liu C, Zhang L, Jia W, Huang W, Li G, Shi J, Wanggou S, Yao K, and Ren C. 2011. Underlying mechanisms for LTF inactivation and its functional analysis in nasopharyngeal carcinoma cell lines. J Cell Biochem 112:1832-1843.

Zhang J, Ling T, Wu H, and Wang K. 2015. Re-expression of Lactotransferrin, a candidate 

oral squamous cell carcinoma cells. J Oral Pathol Med 44:578-584.

418 Zong X YH, Yu Y, Zou D, Ling Z, He X, Meng X. 2011. Possible role of Pax-6 in promoting 419 breast cancer cell proliferation and tumorigenesis. BMB Rep 44:595-600. 
422

423

424

425

426

427

428

429

430

431

432

433

434

435

436

437

438

439

440

441

442

443

444

445

446

447

Figure legends

Figure 1 Top 20 significantly enriched Gene Ontology terms of differentially expressed genes.

Figure 2 Protein-protein interaction networks of differentially expressed genes. Red and green ellipses represent up-regulated and down-regulated genes, respectively. The black borders indicate top 20 up-regulated and down-regulated genes.

Figure 3 Transcription factor-top 20 up-regulated and down-regulated genes network. Diamonds and ellipses represent transcription factors and top 20 up-regulated and downregulated genes, respectively. Red and green ellipses represent up-regulated and down-regulated genes, respectively.

Figure 4 Box plots of seven differentially expressed genes in GSE39262 dataset. The X-axes represent control and case groups while the y-axes represent the relative expression levels of the genes. Seven genes included NETO2, CAMP, METTL7A, TCN1, LTF, CXCL12 and CYP4F3.

Figure 5 Box plots of seven differentially expressed genes in GSE126209 dataset. The X-axes represent control and case groups while the y-axes represent the relative expression levels of the genes. Seven genes included NETO2, CAMP, METTL7A, TCN1, LTF, CXCL12 and CYP4F3.

Figure 6 ROC curves of selected differentially expressed genes in GSE39262 dataset. The xaxes and the $y$-axes show 1-specificity and sensitivity, respectively. $R O C$, receiver operating characteristic. Seven genes included NETO2, CAMP, METTL7A, TCN1, LTF, CXCL12 and CYP4F3.

Figure 7 ROC curves of selected differentially expressed genes in GSE126209 dataset. The $\mathrm{x}$-axes and the $\mathrm{y}$-axes show 1-specificity and sensitivity, respectively. $R O C$, receiver operating characteristic. Seven genes included NETO2, CAMP, METTL7A, TCN1, LTF, CXCL12 and CYP4F3.

Figure S1 The heat map of the top 100 differentially expressed genes. 
Figure 1

Top 20 significantly enriched Gene Ontology terms of differentially expressed genes.

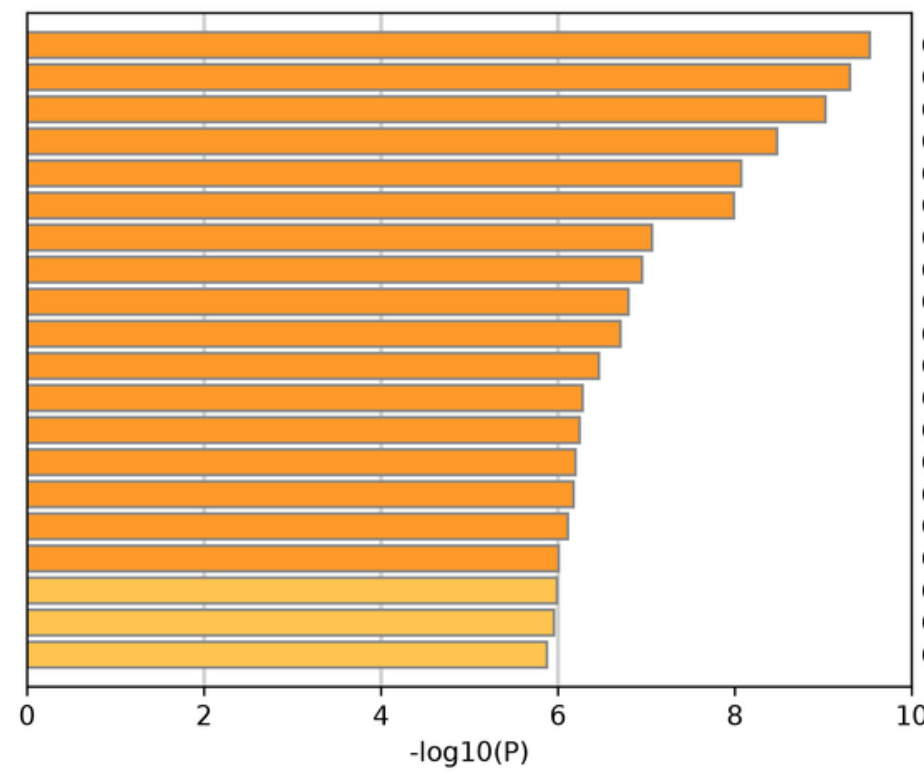

GO:0010942: positive regulation of cell death GO:0002275: myeloid cell activation involved in immune response GO:0045859: regulation of protein kinase activity GO:0051186: cofactor metabolic process GO:0048568: embryonic organ development GO:0001501: skeletal system development GO:0019904: protein domain specific binding GO:0031225: anchored component of membrane GO:0001503: ossification GO:0043009: chordate embryonic development GO:0018212: peptidyl-tyrosine modification GO:0035107: appendage morphogenesis GO:1903827: regulation of cellular protein localization GO:0045444: fat cell differentiation GO:0032787: monocarboxylic acid metabolic process GO:0010638: positive regulation of organelle organization GO:0008134: transcription factor binding GO:0050873: brown fat cell differentiation GO:0033673: negative regulation of kinase activity GO:0031349: positive regulation of defense response 
Figure 2

Protein-protein interaction networks of differentially expressed genes. Red and green ellipses represent up-regulated and down-regulated genes, respectively. The black borders indicate top 20 up-regulated and down-regulated genes.

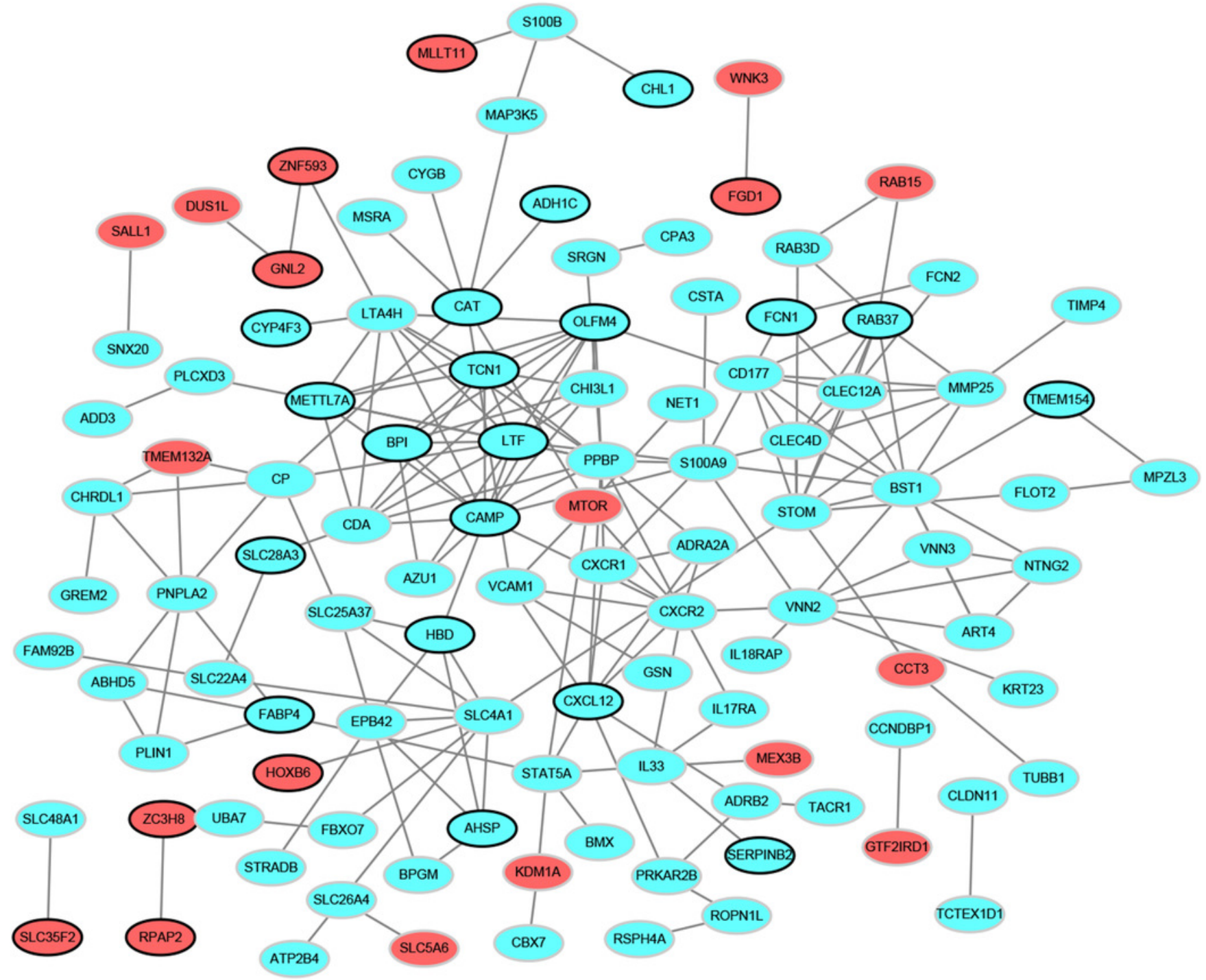


Figure 3

Transcription factor-top 20 up-regulated and down-regulated genes network. Diamonds and ellipses represent transcription factors and top 20 up-regulated and down-regulated genes, respectively. Red and green ellipses represent up-regulated and down-reg

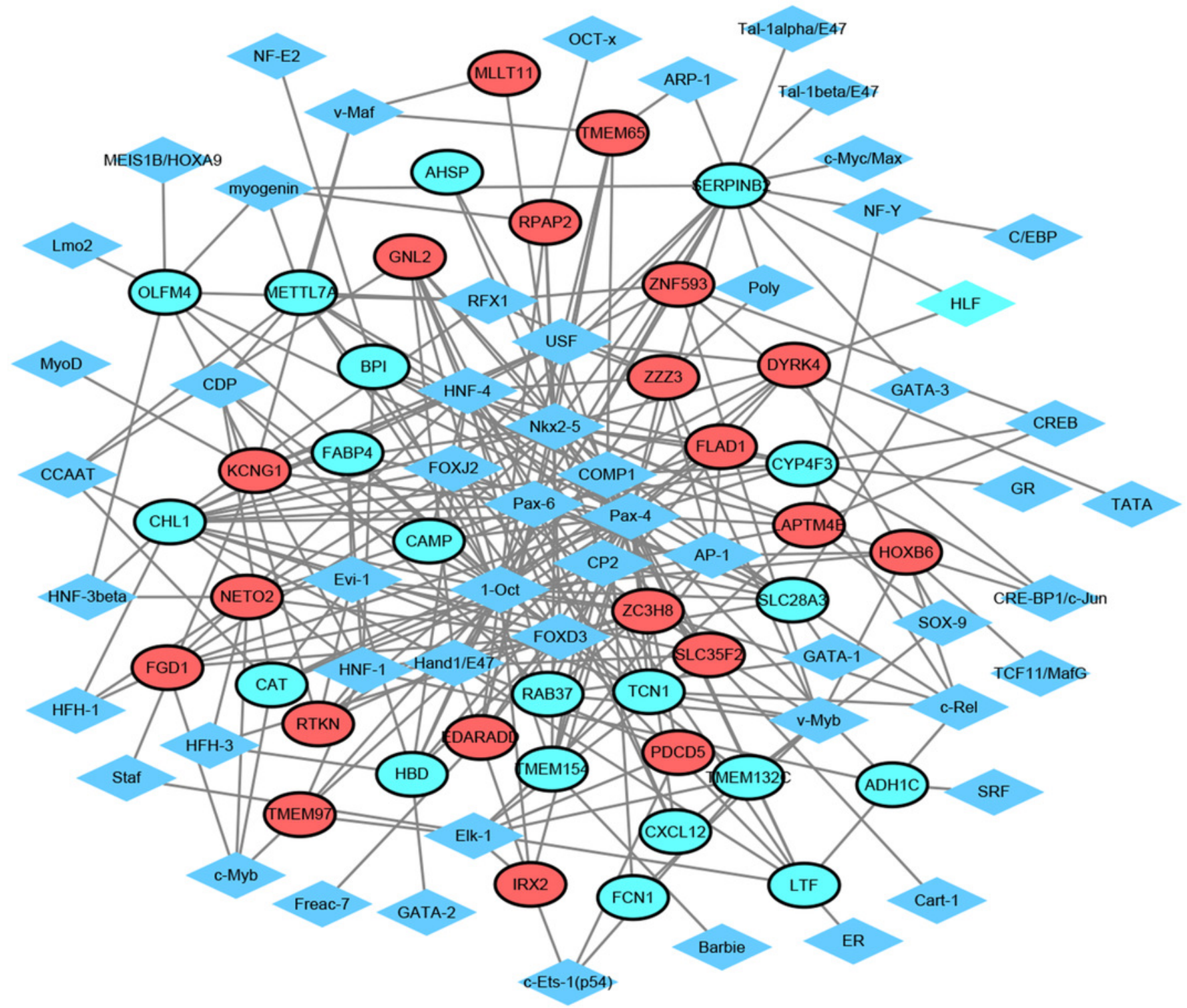


Figure 4

Box plots of seven differentially expressed genes in GSE39262 dataset.

The $x$-axes represent control and case groups while the $y$-axes represent the relative expression levels of the genes. Seven genes included NETO2, CAMP, METTL7A, TCN1, LTF, CXCL12 and CYP4F3.

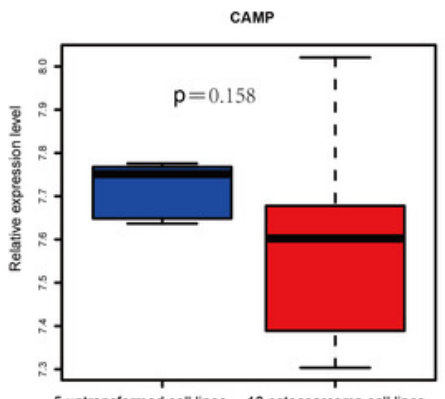

5 untransformed cell lines 10 osteosarcoma cell lines

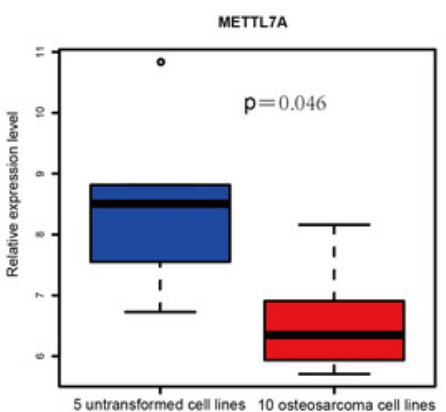

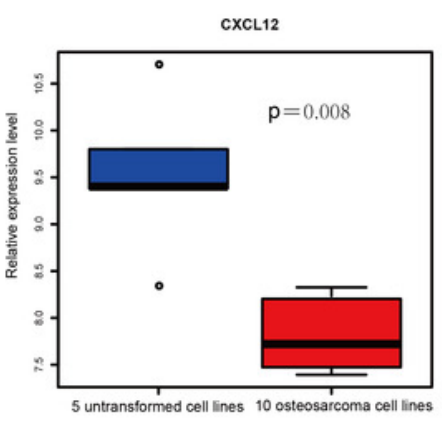
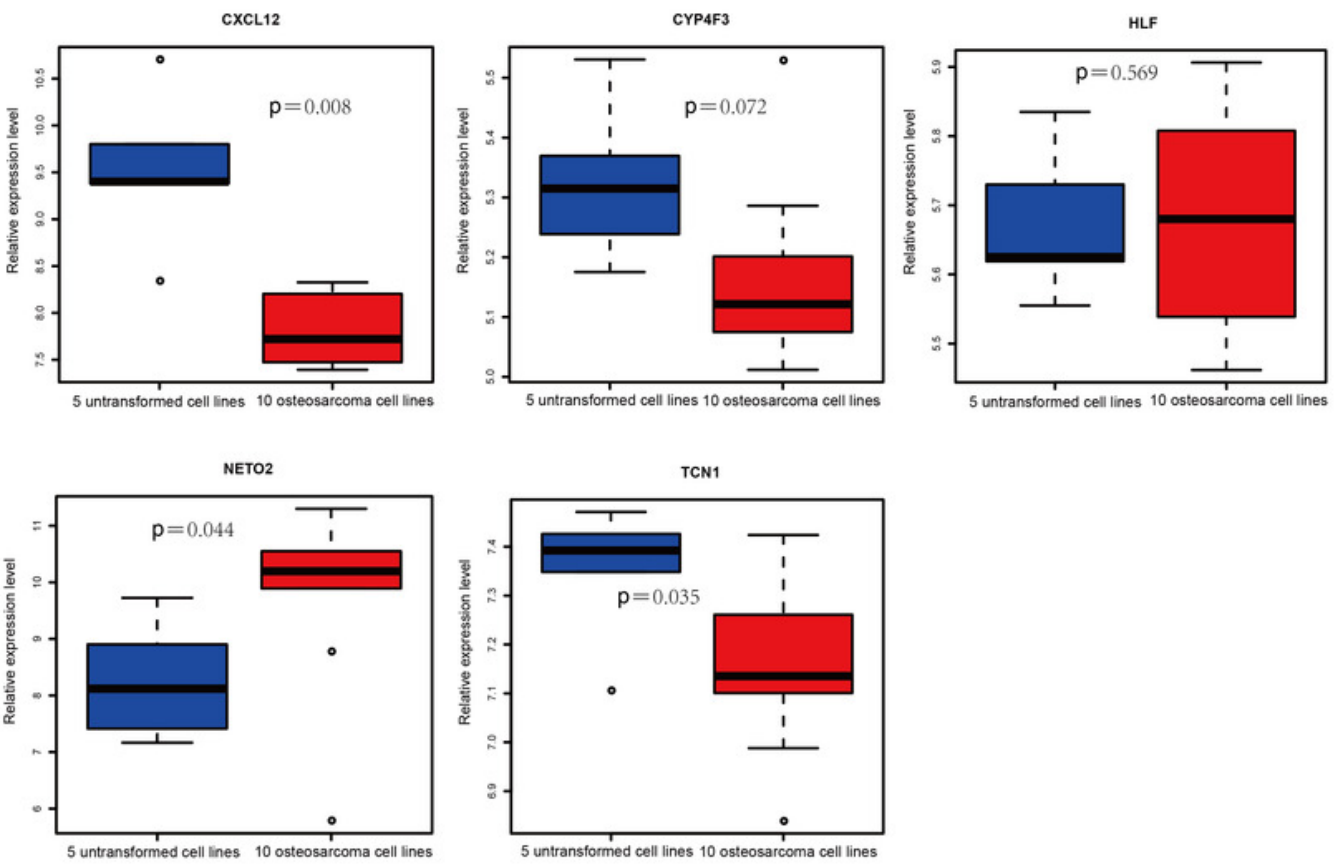
Figure 5

Box plots of seven differentially expressed genes in GSE126209 dataset.

The $x$-axes represent control and case groups while the $y$-axes represent the relative expression levels of the genes. Seven genes included NETO2, CAMP, METTL7A, TCN1, LTF, CXCL12 and CYP4F3.
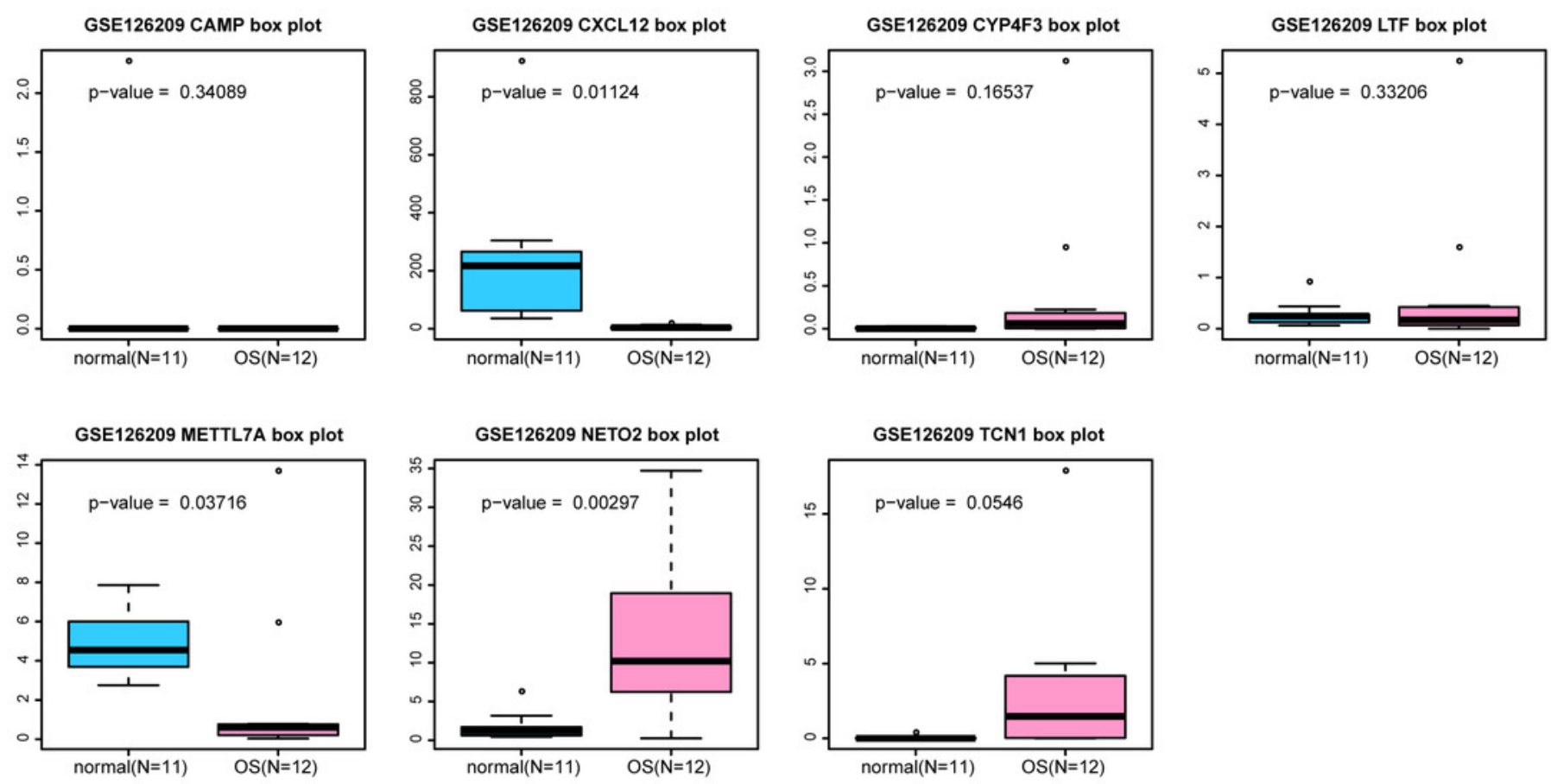
Figure 6

ROC curves of selected differentially expressed genes in GSE39262 dataset.

The $\mathrm{x}$-axes and the $\mathrm{y}$-axes show 1-specificity and sensitivity, respectively. ROC, receiver operating characteristic. Seven genes included NETO2, CAMP, METTL7A, TCN1, LTF, CXCL12 and CYP4F3.
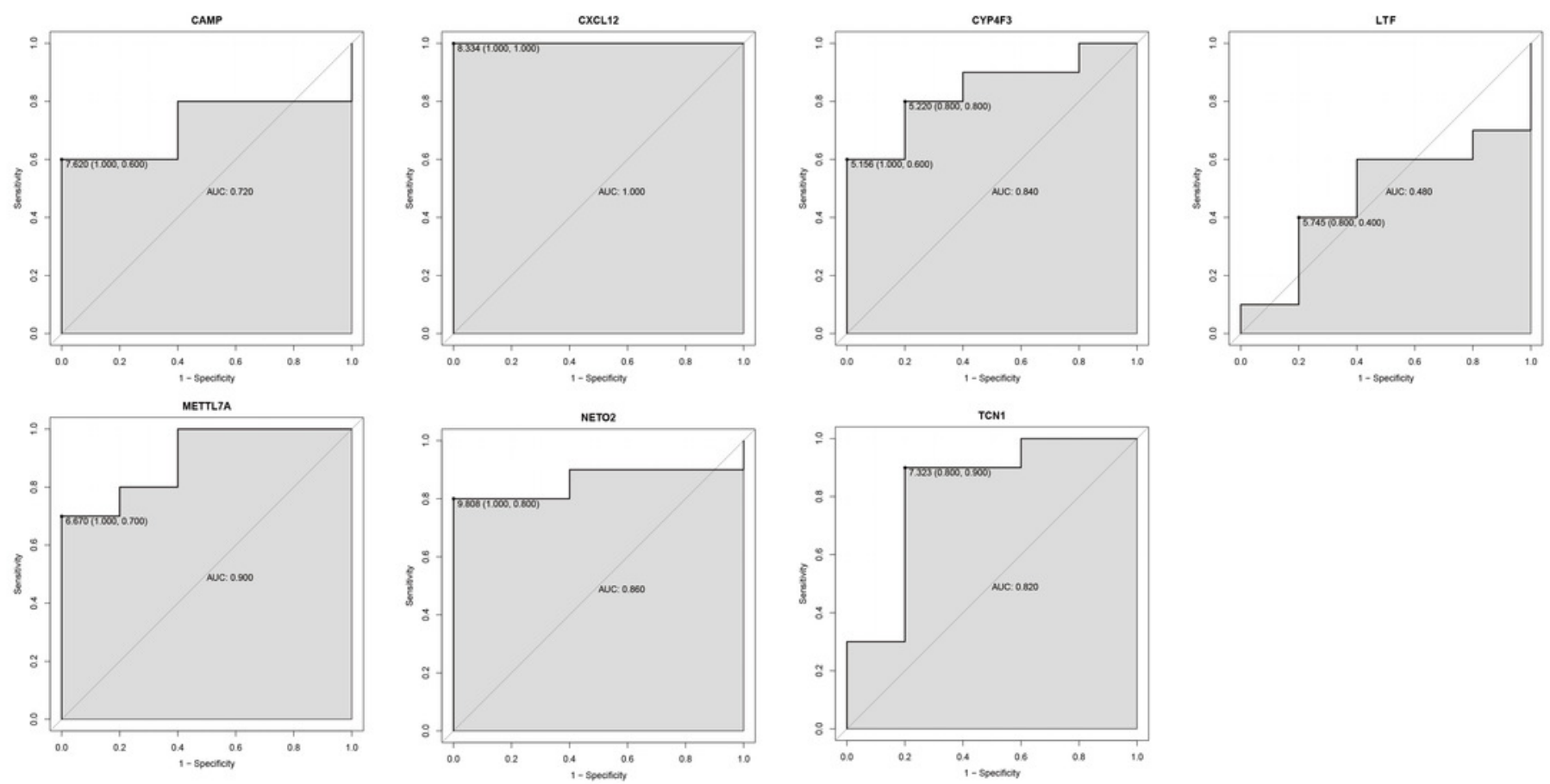
Figure 7

ROC curves of selected differentially expressed genes in GSE126209 dataset.

The $x$-axes and the $y$-axes show 1-specificity and sensitivity, respectively. ROC, receiver operating characteristic. Seven genes included NETO2, CAMP, METTL7A, TCN1, LTF, CXCL12 and CYP4F3.
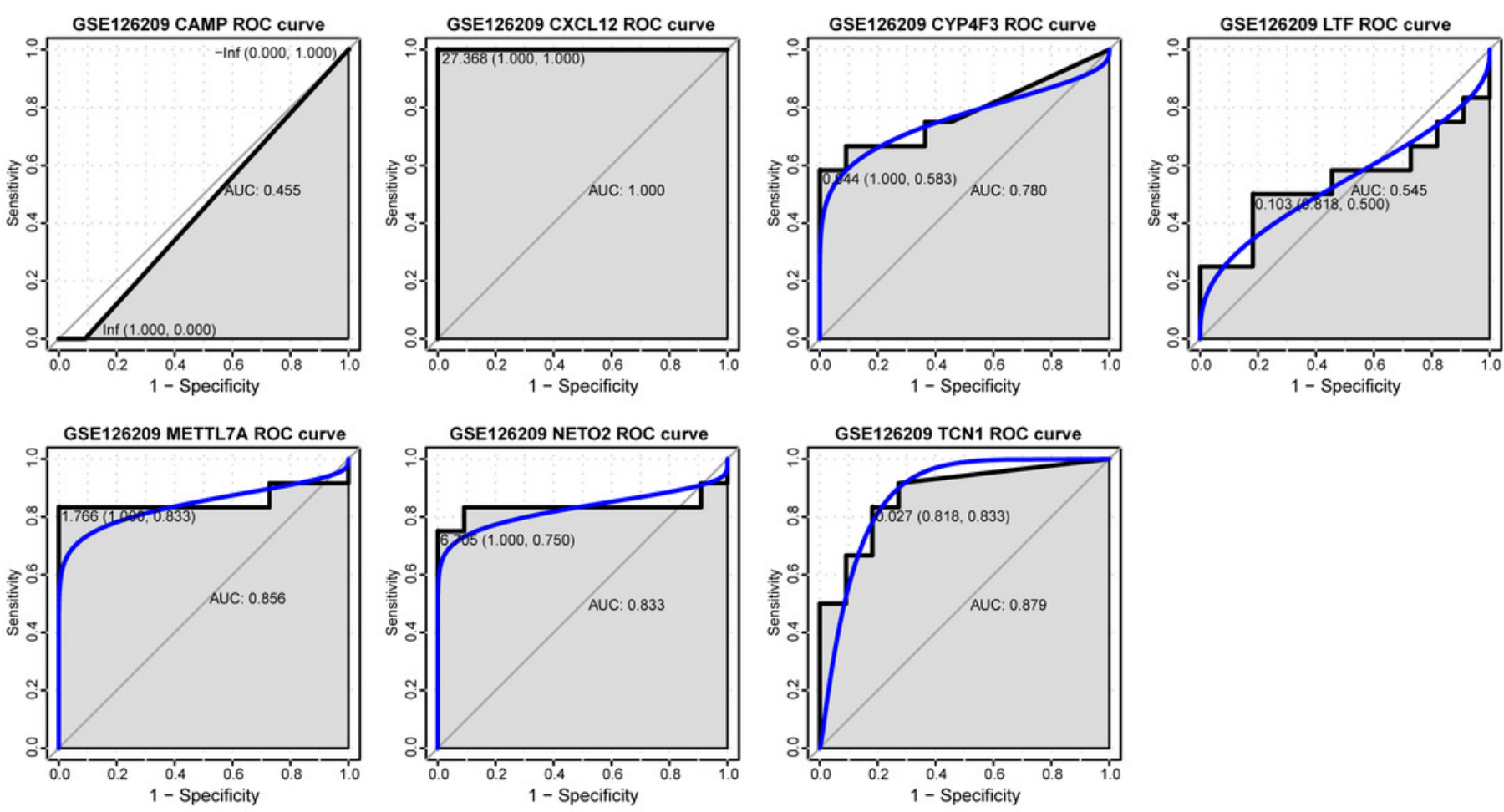


\section{Table $\mathbf{1}$ (on next page)}

The list of top 20 up-regulated and down-regulated differentially expressed genes. 
1 Table 1 The list of top 20 up-regulated and down-regulated differentially expressed genes.

\begin{tabular}{ccrcc} 
Gene Symbol & Combined.ES & \multicolumn{1}{c}{$\boldsymbol{P}_{-}$value } & \multicolumn{1}{c}{ FDR } & Up/Down-regulation \\
\hline NETO2 & 1.773 & $5.48 \mathrm{E}-10$ & $1.16 \mathrm{E}-07$ & Up-regulation \\
RTKN & 1.664 & $3.90 \mathrm{E}-09$ & $6.57 \mathrm{E}-07$ & Up-regulation \\
TMEM65 & 1.669 & $1.08 \mathrm{E}-08$ & $1.54 \mathrm{E}-06$ & Up-regulation \\
MLLT11 & 1.507 & $1.41 \mathrm{E}-08$ & $1.84 \mathrm{E}-06$ & Up-regulation \\
LAPTM4B & 1.499 & $1.78 \mathrm{E}-08$ & $2.21 \mathrm{E}-06$ & Up-regulation \\
ZC3H8 & 1.563 & $1.97 \mathrm{E}-08$ & $2.41 \mathrm{E}-06$ & Up-regulation \\
SLC35F2 & 1.570 & $3.46 \mathrm{E}-08$ & $3.90 \mathrm{E}-06$ & Up-regulation \\
IRX2 & 1.534 & $5.03 \mathrm{E}-08$ & $5.19 \mathrm{E}-06$ & Up-regulation \\
ZNF593 & 1.404 & $7.11 \mathrm{E}-08$ & $6.78 \mathrm{E}-06$ & Up-regulation \\
FLAD1 & 1.451 & $7.54 \mathrm{E}-08$ & $7.10 \mathrm{E}-06$ & Up-regulation \\
KCNG1 & 1.456 & $1.32 \mathrm{E}-07$ & $1.16 \mathrm{E}-05$ & Up-regulation \\
FGD1 & 1.304 & $2.59 \mathrm{E}-07$ & $2.04 \mathrm{E}-05$ & Up-regulation \\
RPAP2 & 1.480 & $2.63 \mathrm{E}-07$ & $2.06 \mathrm{E}-05$ & Up-regulation \\
DYRK4 & 1.405 & $2.83 \mathrm{E}-07$ & $2.20 \mathrm{E}-05$ & Up-regulation \\
EDARADD & 1.360 & $3.06 \mathrm{E}-07$ & $2.33 \mathrm{E}-05$ & Up-regulation \\
PDCD5 & 1.285 & $3.77 \mathrm{E}-07$ & $2.80 \mathrm{E}-05$ & Up-regulation \\
TMEM97 & 1.349 & $4.04 \mathrm{E}-07$ & $2.95 \mathrm{E}-05$ & Up-regulation \\
GNL2 & 1.311 & $4.14 \mathrm{E}-07$ & $3.01 \mathrm{E}-05$ & Up-regulation \\
HOXB6 & 1.527 & $4.47 \mathrm{E}-07$ & $3.17 \mathrm{E}-05$ & Up-regulation \\
ZZZ3 & 1.504 & $5.79 \mathrm{E}-07$ & $3.99 \mathrm{E}-05$ & Up-regulation \\
CAMP & -4.158 & 0 & 0 & Down-regulation \\
AHSP & -3.156 & 0 & 0 & Down-regulation \\
OLFM4 & -3.108 & 0 & 0 & Down-regulation \\
LTF & -3.006 & 0 & 0 & Down-regulation \\
ADH1C & -2.814 & 0 & 0 & Down-regulation \\
\hline & & & & \\
\hline
\end{tabular}




\begin{tabular}{ccccl}
\hline CXCL12 & -2.746 & 0 & 0 & Down-regulation \\
BPI & -2.556 & 0 & 0 & Down-regulation \\
HBD & -2.615 & $8.88 \mathrm{E}-16$ & $5.32 \mathrm{E}-13$ & Down-regulation \\
TCN1 & -2.522 & $1.11 \mathrm{E}-15$ & $6.40 \mathrm{E}-13$ & Down-regulation \\
FABP4 & -2.296 & $7.77 \mathrm{E}-15$ & $4.31 \mathrm{E}-12$ & Down-regulation \\
RAB37 & -2.279 & $2.02 \mathrm{E}-14$ & $1.08 \mathrm{E}-11$ & Down-regulation \\
FCN1 & -2.320 & $2.95 \mathrm{E}-14$ & $1.53 \mathrm{E}-11$ & Down-regulation \\
TMEM154 & -2.279 & $3.13 \mathrm{E}-14$ & $1.55 \mathrm{E}-11$ & Down-regulation \\
METTL7A & -2.181 & $5.44 \mathrm{E}-14$ & $2.47 \mathrm{E}-11$ & Down-regulation \\
CYP4F3 & -2.304 & $6.66 \mathrm{E}-14$ & $2.94 \mathrm{E}-11$ & Down-regulation \\
CAT & -2.109 & $3.44 \mathrm{E}-13$ & $1.43 \mathrm{E}-10$ & Down-regulation \\
CHL1 & -1.974 & $5.51 \mathrm{E}-13$ & $2.17 \mathrm{E}-10$ & Down-regulation \\
TMEM132C & -1.860 & $5.19 \mathrm{E}-12$ & $1.85 \mathrm{E}-09$ & Down-regulation \\
SERPINB2 & -3.082 & $5.64 \mathrm{E}-12$ & $1.97 \mathrm{E}-09$ & Down-regulation \\
SLC28A3 & -2.118 & $8.26 \mathrm{E}-12$ & $2.69 \mathrm{E}-09$ & Down-regulation \\
\hline
\end{tabular}

2 
Table 2 (on next page)

The top 20 significantly enriched KEGG pathways 
1 Table 2 The top 20 significantly enriched KEGG pathways.

\begin{tabular}{|c|c|c|c|c|}
\hline \multirow[t]{2}{*}{ ID } & Term & $P_{-}$value & Count & Gene Symbols \\
\hline & Regulation of lipolysis in & & & ADRB2, FABP4, PDE3B, PIK3CD, PLIN1, PTGER3, IRS2, ABHD5, \\
\hline \multirow[t]{3}{*}{ hsa04923 } & adipocytes & 0.0001 & 10 & $P N P L A 2, A D C Y 4$ \\
\hline & & & & BAG1, EIF2S1, HSPA2, LMAN1, MAN1A1, MAP3K5, P4HB, EIF2AK2, \\
\hline & Protein processing in endoplasmic & & & RPN2, RRBP1, SSR2, PREB, SEC24A, SEC61G, UBQLN2, DNAJB11, \\
\hline \multirow[t]{5}{*}{ hsa04141 } & reticulum & 0.0001 & 19 & UBQLN4, DNAJC1, UBE2J2 \\
\hline & & & & $\begin{array}{l}\text { BCL2L1, CASP8, CDKN2A, CEBPA, CKS1B, COL4A1, DVL1, E2F1, } \\
\text { EPAS1, FGF7, FGF13, FLT3, MTOR, GLI2, GLI3, GNAQ, PIK3CD, PTEN, }\end{array}$ \\
\hline & & & & PTGER2, PTGER3, RARB, RXRA, CXCL12, SKP2, STAT5A, TGFB3, \\
\hline & & & & TGFBR2, VEGFA, NCOA4, FGF16, PIAS2, ARHGEF11, LEF1, ADCY4, \\
\hline & & & & SHC1, CALM1, CHEK1, ELK1, FDPS, GPS2, MSX2, RANBP1, TLN1, \\
\hline \multirow[t]{2}{*}{ hsa05200 } & Pathways in cancer & 0.0002 & 34 & $V C A M 1, T L N 2$ \\
\hline & & & & CTH, ENO1, GAPDH, PC, SHMT1, TALDO1, TKT, TPI1, SDS, RPIA, \\
\hline \multirow[t]{2}{*}{ hsa01230 } & Biosynthesis of amino acids & 0.0004 & 11 & $A A D A T, C A T, M D H 2, M E 1, M C E E$ \\
\hline & & & & COL2A1, COL4A1, COL5A2, COL10A1, COL11A1, COL17A1, CPA3, \\
\hline \multirow[t]{2}{*}{ hsa04974 } & Protein digestion and absorption & 0.0019 & 11 & SLC7A8, COL18A1, COL27A1, SLC16A10 \\
\hline & & & & CALM1, MAPK14, MAP3K5, NFKBIE, NTF3, NTRK2, PIK3CD, PRKCD, \\
\hline hsa04722 & Neurotrophin signaling pathway & 0.0022 & 13 & RPS6KA2, SHC1, MAGED1, PRDM4, IRAK3 \\
\hline
\end{tabular}


CPT1A, ELAVL1, MTOR, LEP, PIK3CD, PPP2R5A, PPP2R5D, PRKAB2, IRS2, CREB5, CAMKK2, STRADB, CREB3L1, PRKCD, PRKCE, PTEN, hsa04152 AMPK signaling pathway 0.0023 RPS6KA2, TBC1D4, JAK2, NFKBIE, RXRA, SOCS2, CAT, ADCY4, CALM1, Pentose phosphate pathway, nonoxidative phase, fructose $6 \mathrm{P}=>$

\section{M00007 ribose 5P \\ hsa03060 Protein export}

AGE-RAGE signaling pathway in hsa04933 diabetic complications

hsa04360 Axon guidance

0.0045

16
TALDO1, TKT, RPIA

OXA1L, SRP54, SRP72, SEC61G, SRPRB

COL4A1, MAPK14, JAK2, PIK3CD, PRKCD, PRKCE, STAT5A, TGFB3, TGFBR2, VCAM1, VEGFA, CD247, MTOR, NFKBIE, RXRA, STAT6, 11 IL27RA, LHB, SHC1, SOCS2, AOX1, BCL2L1, IL5RA, LEP, PIAS2 EFNA1, EFNA3, EFNA5, EFNB1, EPHA3, EPHB2, EPHB6, FES, PIK3CD, CXCL12, SEMA7A, SEMA3A, RHOD, SEMA4C, NTNG2, PLXNA4 CALM1, MAPK14, EFNA1, EFNA3, EFNA5, FGF7, FGF13, GNAQ, ITGB3, PFN2, PIK3CD, TLN1, VEGFA, FGF16, RAPGEF3, APBB1IP, TLN2, ADCY4, BCL2L1, COL2A1, COL4A1, MTOR, ITGA7, JAK2, PPP2R5A, hsa04015 Rap1 signaling pathway 0.0053 18 PPP2R5D, PTEN, RXRA, TLR4, CREB5, CREB3L1, THEM4 ALOX15, GGT5, GPX3, GPX7, LTA4H, CYP4F3, PLA2G5, PTGES, GSTA4, hsa00590 Arachidonic acid metabolism $0.0055 \quad 8 \quad$ GSTM3, SRM, GGCT 


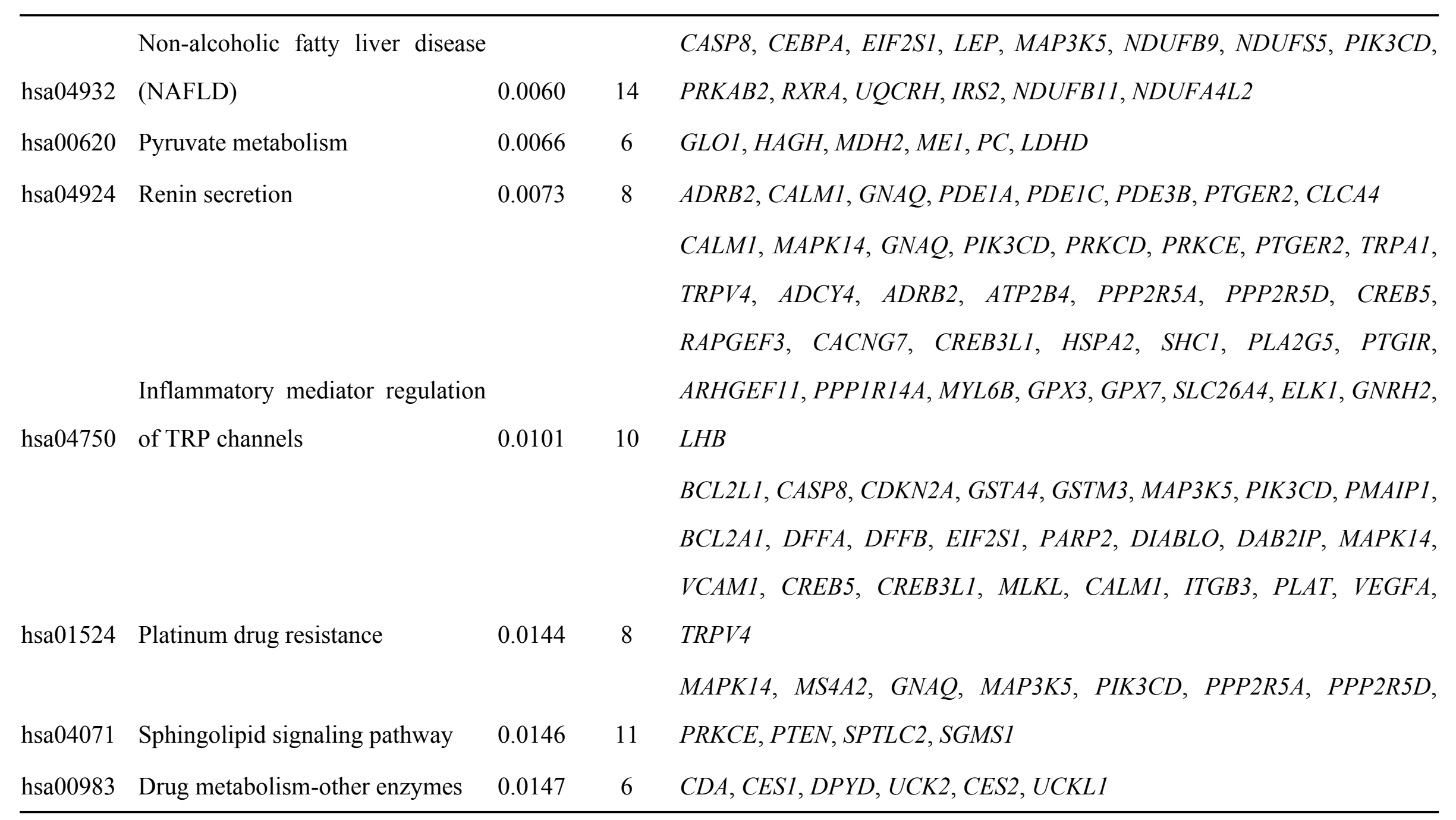

$3 K E G G$ Kyoto Encyclopedia of Genes and Genomes 
Table 3 (on next page)

The top 6 TF that has the most downstream genes 
Table 3 The top 6 TF that has the most downstream genes.

\begin{tabular}{|c|c|c|}
\hline TF & Number* & Gene Symbol \\
\hline Pax-4 & 31 & $\begin{array}{l}\text { FLAD1, ZZZ3, GNL2, EDARADD, RTKN, LTF, CAMP, CHL1, ADH1C, TMEM154, IRX2, FABP4, LAPTM4B, } \\
\text { SLC28A3, FGD1, CXCL12, HBD, TCN1, CAT, SLC35F2, DYRK4, METTL7A, OLFM4, AHSP, RAB37, TMEM97, } \\
\text { SERPINB2, CYP4F3, PDCD5, BPI, KCNG1 }\end{array}$ \\
\hline $1-\mathrm{Oct}$ & 22 & $\begin{array}{l}\text { FLAD1, GNL2, RPAP2, EDARADD, ZC3H8, CHL1, CAMP, IRX2, FABP4, TMEM65, SLC28A3, HBD, TCN1, CAT, } \\
\text { TMEM132C, DYRK4, METTL7A, TMEM97, HOXB6, SERPINB2, BPI, KCNG1 }\end{array}$ \\
\hline Nkx2-5 & 19 & $\begin{array}{l}\text { FLAD1, MLLT11, RPAP2, EDARADD, CAMP, CHL1, TMEM154, LAPTM4B, TMEM65, SLC28A3, FGD1, TCN1, } \\
\text { SLC35F2, DYRK4, AHSP, SERPINB2, CYP4F3, KCNG1, BPI }\end{array}$ \\
\hline HNF-4 & 16 & $\begin{array}{l}\text { ZZZ3, ZNF593, GNL2, LTF, CHL1, CAMP, LAPTM4B, SLC28A3, FGD1, CXCL12, HBD, SLC35F2, METTL7A, } \\
\text { RAB37, CYP4F3, KCNG1 }\end{array}$ \\
\hline FOXD3 & 14 & $\begin{array}{l}\text { GNL2, RPAP2, EDARADD, ZC3H8, CHL1, LTF, FABP4, FGD1, HBD, DYRK4, METTL7A, OLFM4, NETO2, } \\
\text { RAB37 }\end{array}$ \\
\hline Pax-6 & 13 & $\begin{array}{l}\text { EDARADD, GNL2, CHL1, TMEM65, SLC28A3, CXCL12, HBD, TCN1, DYRK4, NETO2, SERPINB2, CYP4F3, } \\
\text { KCNG1 }\end{array}$ \\
\hline
\end{tabular}

2

3 Number* indicates the number of genes regulated by the TF. TF transcription factor 